MRS Advances (C) 2017 Materials Research Society

DOI: $10.1557 /$ adv.2017.83

\title{
Modeling of Plasma Expansion during Pulsed Electron Beam Ablation of Graphite
}

\author{
Muddassir Ali and Redhouane Henda \\ Bharti School of Engineering, Laurentian University, 935 Ramsey Lake Road, Sudbury, ON P3E \\ 2C6, Canada.
}

\begin{abstract}
Pulsed electron beam ablation (PEBA) has proven to be a promising and powerful technique for the growth of high quality thin films. Pulsed electron beam film deposition consists of many physical processes including target material heating, target ablation, plasma plume expansion, and film growth on a substrate. Plasma plume expansion into a vacuum or an ambient gas is a fundamental issue in PEBA as the quality of thin films deposited onto the substrate depends on the composition, energy and density of particles ejected from the target. In the present study, gas-dynamics equations are solved to investigate plasma expansion induced by interaction of a nanosecond electron beam pulse $(\sim 100 \mathrm{~ns})$ with a graphite target in an argon atmosphere at reduced pressure. The spatio-temporal profiles of the temperature, pressure, velocity, and density of the plasma plume are numerically simulated for a beam efficiency of 0.6 and accelerating voltage of $15 \mathrm{kV}$. The preliminary results show a rich variety of behaviors. The model is validated by comparing some of the obtained simulation results with experimental data available in the literature.
\end{abstract}

\section{INTRODUCTION}

One of the key developments in pulsed energy processing techniques of materials is the emergence of pulsed electron beam ablation (PEBA) for thin film growth [1,2]. Pulsed electron beam ablation has been recognized as an effective, powerful and promising technique for the fabrication of thin films and structures of high quality. PEBA offers numerous advantages over other conventional techniques, e.g., versatile setup, congruent material transfer from bulk to film under optimum conditions, low cost, and small footprint. One of the most attractive features of PEBA is the possibility of fabricating films under non-thermal conditions [1-3]. During PEBA, an intense electron pulse $(\sim 100 \mathrm{~ns})$ strikes the target surface, triggering a sequence of strongly coupled processes. Electron beam radiation is absorbed by the target surface inducing ablation (heating and vaporization of a target surface) and expansion of a plasma plume just above the impinged target surface. The plasma, consisting of ablated particles, expands with a supersonic velocity in the ambient atmosphere towards a properly placed substrate relatively to the target.

Despite PEBA increasing technological significance, details of the complex processes responsible for its characteristic features are not well explored. The processes controlling PEBA are strongly coupled and nonlinear. For a better understanding of phenomena affecting the quality of thin film growth, it is expedient to develop a comprehensive model of the process. Material ablation, plasma expansion, and the interaction of plume particles with an ambient gas are critical in defining the morphology and structure of thin films. The properties of the expanding plume play a crucial role in defining the ultimate properties of the deposited films [4]. The distribution and range of the kinetic energy of the ablated plume particles significantly affect the quality of the deposited film. The presence of an ambient gas influences both the energy and nature of ablated particles impinging on the substrate surface, which, in turn, govern the properties of the deposited films [5]. Accordingly, modeling of the plasma plume expansion into 
an ambient gas is important not only to acquire a better understanding of the phenomena involved, but also to optimize and eventually control PEBA process. To our knowledge, no attempt has been made to address the foregoing discussed issues in PEBA. In the present work, a mathematical model is presented to study plasma expansion induced upon irradiation with a nanosecond ( $100 \mathrm{~ns})$ polyenergetic pulsed electron beam (for a beam efficiency of 0.6 and accelerating voltage of $15 \mathrm{kV}$ ) from a graphite target placed in an argon atmosphere at reduced pressure. One-dimensional gas-dynamics equations are employed and numerically solved for the entire physical domain involved.

\section{MATHEMATICAL MODEL}

\section{Target ablation}

Upon irradiation with a pulsed electron beam, the surface of the target goes through heating and phase change from the solid phase to the vapor phase [6]. Accordingly, phase transition must be taken into account during target ablation. Target heating and vaporization can be defined by a two-stage, one-dimensional heat conduction equation. The model describing heating and vaporization induced during pulsed electron beam interaction with a graphite target has been discussed in detail elsewhere [7].

\section{Plasma expansion dynamics}

Under the prevailing conditions of our study, viz., plasma expansion during nanosecond pulsed electron beam ablation, the Knudsen number is much smaller than 0.01 [8], and, as such, the application of Navier-Stokes equations is warranted. The expansion dynamics of the electron beam-induced plasma plume into an ambient gas can be described by a set of one-dimensional compressible Navier-Stokes equations of gas-dynamics expressing mass, momentum and energy conservation as [9],

$\frac{\partial \rho}{\partial t}+\nabla \cdot(\rho \overrightarrow{\boldsymbol{u}})=0$

$\frac{\partial \rho \overrightarrow{\boldsymbol{u}}}{\partial t}+(\nabla \cdot \rho \overrightarrow{\boldsymbol{u}} \overrightarrow{\boldsymbol{u}})+\nabla P+\nabla \cdot \overline{\bar{\tau}}=0$

$\frac{\partial \rho E}{\partial t}+(\nabla \cdot \rho E \overrightarrow{\boldsymbol{u}})+\nabla \cdot P \overrightarrow{\boldsymbol{u}}+\nabla \cdot q+\nabla \cdot(\overrightarrow{\boldsymbol{u}} \cdot \overline{\bar{\tau}})=0$

where $\rho, \overrightarrow{\boldsymbol{u}}$, and, $P$ are the plasma density, velocity, and pressure, respectively, $\overline{\bar{\tau}}$ is the viscous shear stress tensor, and $q$ is the heat flux due to conduction. The total specific energy of the plasma, $E$, is expressed in terms of the specific internal energy, $\mathcal{E}$, and kinetic energy as,

$E=\mathcal{E}+\frac{\overrightarrow{\boldsymbol{u}}^{2}}{2}$

Assuming that the expanding plasma plume follows the ideal gas law (under reduced pressure), the specific internal energy and pressure of plasma can be given by,

$\rho \mathcal{E}=n\left[\frac{3}{2}\left(1+x_{e}\right) k_{B} T+\sum_{i=1}^{n}\left(I P_{i} \sum_{i=1}^{n} x_{i}\right)\right]$

$P=\left(1+x_{e}\right) n k_{B} T$ 
where $n=\rho / m$ is the number density of the plasma, $x_{e}$ is the partial fraction of electrons in the ablated plume, $T$ is the temperature of the plasma, $I P_{i}$ is the ionization energy of carbon at level $i$, $x_{i}$ is the partial fraction of charged ions, and $k_{B}$ is the Boltzmann constant. Under our present process conditions the local thermodynamic equilibrium approximation (LTE) is reasonable as per McWhirter criterion [10]. When particles in the ablated plume reach LTE, the ionization state of the plasma can be calculated by the Saha-Eggert equation [11],

$\frac{x_{i} x_{e}}{x_{i-1}}=2 \frac{g_{i}}{g_{i-1}} \frac{1}{n}\left(\frac{2 \pi m_{e} k_{B} T}{h^{2}}\right)^{3 / 2} \exp \left(-\frac{I P_{i}}{k_{B} T}\right)$

where $g_{i}$ is the degeneracy factor of an ionized atom at level $i, m_{e}$ is the mass of an electron, and $h$ is the Planck constant.

The set of equations (1-3) are subjected to initial and boundary conditions as follows:

$T=300 \mathrm{~K} ; \rho=0 ; u=0 \mathrm{~m} / \mathrm{s} \quad$ at $t=0$

The boundary conditions at the interface between the target and ambient gas are based on the data calculated from the Knudsen layer (KL) theory [12]. Accordingly, and for a back flux coefficient of 0.18 , the following expressions are used for the outer edge of the Knudsen layer,

$T_{k}=0.67 T_{s} ; \quad \rho_{k}=0.31 \rho_{s} ; \quad u_{k}=M \sqrt{\gamma_{v} k_{B} T_{k} / m}$

where $T_{k}, \rho_{k}$, and $u_{k}$ are the temperature, density, and velocity of the particles leaving the Knudsen layer, respectively. $T_{s}, \rho_{s}$, and $P_{S}$ are the temperature, density, and pressure at the target surface, respectively, and $\gamma_{v}$ is the specific heat ratio of vapor. The boundary conditions are,

$T=T_{k} ; \rho=\rho_{k} ; u=u_{k} \quad$ at $x=0$

Plasma properties have been calculated from relationships and expressions given in Chen and Bogaerts [13]. Equations (1-10) have been set up and solved using COMSOL Multiphysics ${ }^{\circledR}$. We have refined the space mesh and time step until the calculation data have become independent of both.

\section{RESULTS AND DISCUSSION}

The spatio-temporal evolutions of the temperature, velocity, pressure, and electron density of expanding plasma induced during PEBA of graphite are discussed in the following. Results from our recent investigations have indicated that the optimum values of beam efficiency and accelerating voltage are about 0.6 and $15 \mathrm{kV}$, respectively, for the target to reach optimal ablation performance $[7,14]$. The results correspond to a beam efficiency of 0.6 and accelerating voltage of $15 \mathrm{kV}$. The plasma plume temperature is illustrated in Figure 1. A rich variety of shock waves can be clearly observed from the form of the temperature profiles at different times during the beam pulse. As can be observed in Figure 1, the plasma temperature increases rapidly in the vicinity of the target surface. At $20 \mathrm{~ns}$ (onset of ablation), the plasma temperature can reach a maximum value of $11,800 \mathrm{~K}$ at $\sim 0.5 \mathrm{~cm}$ above the target surface. Between one-third and two-thirds of the beam pulse, inner and outer shock waves can be clearly observed, see Figure 1, $\mathrm{t}=40 \mathrm{~ns}, 60 \mathrm{~ns}$. 'Doubling' of the shock wave is likely the result of the expanding plasma being pushed backward by the ambient gas. The plasma temperature seems to decrease, albeit only slightly, e.g., $\sim 11,400 \mathrm{~K}$ at $40 \mathrm{~ns}$ and $\sim 11,300 \mathrm{~K}$ at $60 \mathrm{~ns}$ (see Figure 1), relatively to the plasma during the early stage of the pulse. This is the result of energy losses owing to internal interactions within the expanding plasma and plasma interaction with the ambient gas. Towards the end of the pulse, the shock waves seem to blend together resulting in a broader shock wave, 
as depicted in Figure 1 at $\mathrm{t}=80 \mathrm{~ns}, 100 \mathrm{~ns}$. The plasma temperature slightly increases due to the polyenergetic nature of the electron beam $[14,15]$.

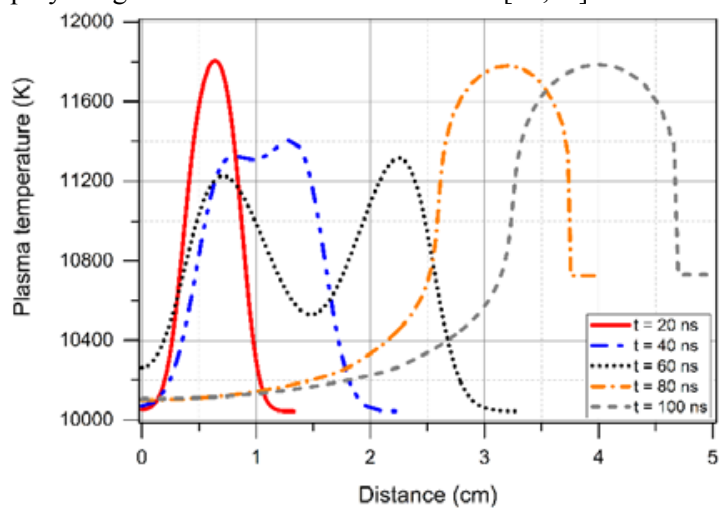

Figure 1. Spatial distribution of plasma temperature at various time intervals.

Plasma velocity profile calculated for various time intervals is shown in Figure 2. As the plume expands, the plasma velocity increases significantly over the pulse duration. For example, at $20 \mathrm{~ns}$ corresponding to the onset of ablation, the plasma velocity is just below $5000 \mathrm{~m} / \mathrm{s}$. For larger values of time, the plume velocity further increases as the plume expands in the upward direction and reaches a maximum value of $\sim 9900 \mathrm{~m} / \mathrm{s}$ at the shock wave peak at the end of the pulse, see Figure 2, $t=100 \mathrm{~ns}$. As anticipated, the presence of the low pressure ambient gas slightly affects the plume velocity and, in turn, allows the plume to expand over a large distance.

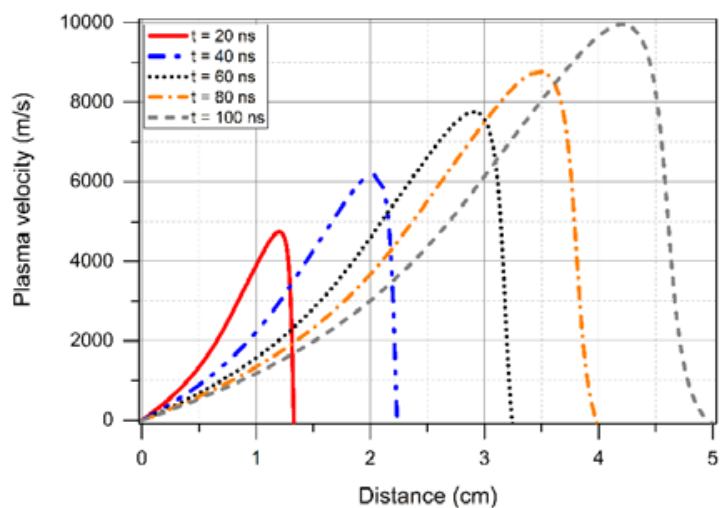

Figure 2. Spatial distribution of plasma velocity at various time intervals.

The spatial distribution of the plume pressure as a function of time is presented in Figure 3. It can be observed that, at first, the expanding plasma encounters an isentropic rarefaction wave, where the pressure decreases as the plasma plume moves forward. As anticipated, the maximum 
pressure is located near the target surface. As it can be observed, at $20 \mathrm{~ns}$, the maximum value of the plasma pressure is $\sim 2.7 \times 10^{6} \mathrm{~Pa}$, which decreases as the plasma plume moves away from the surface. As time progresses, the pressure of expanding plasma declines. At the end of beam pulse $(100 \mathrm{~ns})$, the plume pressure reaches a maximum of $4 \times 10^{5} \mathrm{~Pa}$ only in the vicinity of the target surface (about eightfold less than at $20 \mathrm{~ns}$ ).

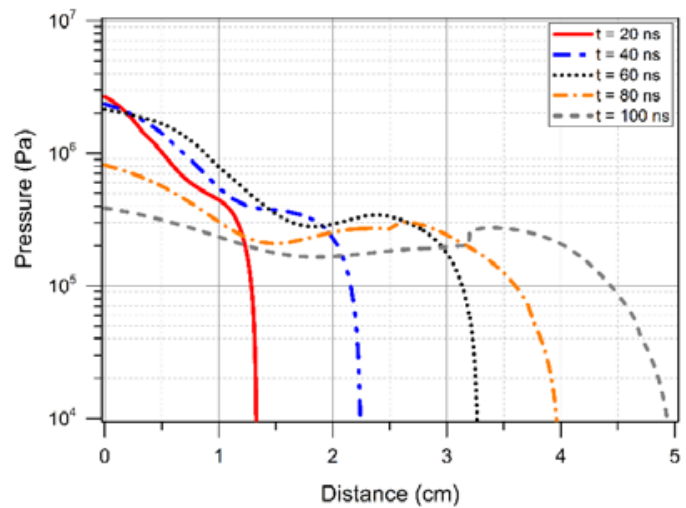

Figure 3. Spatial distribution of plasma pressure at various time intervals.

Figure 4 shows the spatial distribution of the electron density of the plasma at various time intervals. It can be observed that the electron density exhibits a maximum, which moves away from the target surface as time increases. At $20 \mathrm{~ns}$, the maximum value of electron density is $\sim 5.2 \times 10^{23} \mathrm{~m}^{-3}$, which is located at $\sim 0.5 \mathrm{~cm}$ from the target surface. For longer times, such as at $80 \mathrm{~ns}$, the maximum electron density is $2.5 \times 10^{23} \mathrm{~m}^{-3}$ and located $\sim 2.8 \mathrm{~cm}$ from the target surface. Overall, the electron density and plasma pressure profiles seem to show similar structures.

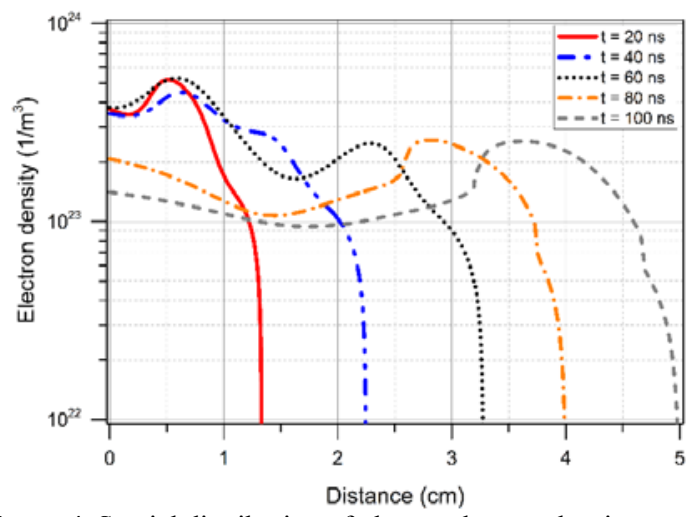

Figure 4. Spatial distribution of plasma electron density at various time intervals. 


\section{MODEL ASSESSMENT}

In order to assess the model, some of the calculated variables from the current model have been compared with experimental data available in the literature for plasma induced during PEBA [15-17]. Experimental results have reported electron density values in the range of $10^{22}$ $10^{23} \mathrm{~m}^{-3}$ [15-17]. This is in accordance with our calculations, whereby the average electron density is $\sim 10^{23} \mathrm{~m}^{-3}$. It has been found that the average temperature of the plasma varies from 1 $\mathrm{eV}$ to $1.25 \mathrm{eV}[15,16]$. The results based on our calculations indicate that the plasma temperature is $\sim 1 \mathrm{eV}$, which is in quantitative agreement with the experimental data. Finally, it has been reported that the propagation of the plasma plume can reach $\sim 10^{4} \mathrm{~m} / \mathrm{s}$ [17]. This is in good agreement with our calculated plasma velocity of $9900 \mathrm{~m} / \mathrm{s}$ as depicted in Figure 2.

\section{CONCLUSION}

We have proposed a one-dimensional gas-dynamics model based on Navier-Stokes equations to investigate the properties of plasma expansion induced during PEBA. Model calculations allow the estimation of the spatio-temporal dependence of the temperature, velocity, pressure, and electron density of the expanding plasma. The calculated preliminary results are in good agreement with experimental data reported in the literature.

\section{ACKNOWLEDGMENTS}

This work was supported by the Natural Sciences and Engineering Research Council (NSERC) of Canada.

\section{REFERENCES}

1. R.M. Gilgenbach, S.D. Kovaleski, J.S. Lash, L.K. Ang and Y.Y. Lau, IEEE Trans. Plasma Sci. 27, 150-158 (1999).

2. G. Müller, M. Konijnenberg, G. Kraff and C. Schultheiss, in Science and Technology of Thin Films, edited by F.C.Matacotta and G. Ottaviani (World Scientific, Singapore, 1995), pp. 89-119.

3. S.D. Kovaleski, R.M. Gilgenbach, L.K. Ang, Y.Y. Lau and J.S. Lash, Appl. Surf. Sci. 127129, 947-952 (1998).

4. A. Misra, A. Mitra and R.K. Thareja, Appl. Phys. Lett. 74, 929-931 (1999).

5. R.K. Thareja, R.K. Dwivedi and Abhilasha, Phys. Rev. B 55, 2600-2605 (1999).

6. M. Strikovski and K.S. Harshavardhan, Appl. Phys. Lett. 82, 853-855 (2003).

7. M. Ali and R. Henda, ECS J. Solid State Sci. Technol. 4, P369-P375 (2015).

8. A. Faghri, and Y. Zhang, Transport Phenomena in Multiphase Systems, 1st ed. (Academic Press, Burlington, 2006).

9. R.B. Bird, W.E. Stewart and E.N. Lightfoot, Transport Phenomena, 2nd ed. (John Wiley and Sons Inc., New York, 2002).

10. G. Cristoforetti, A.D. Giacomo, M. Dell'Aglio, S. Legnaioli, E. Tognoni, V. Palleschi, and N. Omenetto, Spectrochimica Acta Part B: Atomic Spectroscopy 65, 86-95 (2010).

11. Y.B. Zel'dovich and Y.P. Raizer, Physics of Shock Waves and High-Temperature Hydrodynamic Phenomena, vol. 1 (Academic Press Inc., New York, 1966).

12. C. J. Knight, AIAA J., 17, 519-523 (1979).

13. Z. Chen and A. Bogaerts. J. Appl. Phys. 97, 063305 (2005). 
14. M. Ali and R. Henda, Appl. Surf. Sci. 396, 67-77 (2017).

15. T. Witke, A. Lenk and B. Schultrich, IEEE Trans. Plasma Sci. 24, 61-62 (1996).

16. S. Tricot, C.B. Leborgne, M. Nistor, E. Millon and J. Perrière, J. Phys. D: Appl. Phys. 41, 175205 (2008).

17. M. Nistor, F. Gherendi and N.B. Mandache, IEEE Trans. Plasma Sci. 39, 2800-2801 (2011). 